\title{
Confinining properties of QCD in strong magnetic backgrounds
}

\author{
Claudio Bonati $i^{1,2, a}$, Massimo D’Elia ${ }^{1,2, b}$, Marco Mariti ${ }^{1,2, c}$, Michele Mesiti ${ }^{1,2, d}$, Francesco \\ Negro $^{2, e}$, Andrea Rucci ${ }^{1,2, f}$, and Francesco Sanfilippo ${ }^{3, g}$ \\ ${ }^{1}$ Dipartimento di Fisica dell'Università di Pisa, Largo Pontecorvo 3, I-56127 Pisa, Italy \\ ${ }^{2}$ INFN sezione di Pisa, Largo Pontecorvo 3, I-56127 Pisa, Italy \\ ${ }^{3}$ School of Physics and Astronomy, University of Southampton, SO17 1BJ Southampton, United Kingdom
}

\begin{abstract}
Strong magnetic backgrounds are known to modify QCD properties at a nonperturbative level. We discuss recent lattice results, obtained for $N_{f}=2+1$ QCD with physical quark masses, concerning in particular the modifications and the anisotropies induced at the level of the static quark-antiquark potential, both at zero and finite temperature.
\end{abstract}

\section{Introduction}

The effects of strong magnetic fields on the properties of strongly interacting matter are relevant to many phenomenological contexts, ranging from heavy ion collisions [1-6] to cosmology [7, 8] and magnetars [9]. For that reason, the issue has been the subject of many recent theoretical studies (for a review, see Refs. [10,11]). Quarks are directly coupled to magnetic fields, through their electric charges, however many interesting effects emerge also in the gluon sector, mediated by quark loops. Lattice QCD simulation have proved to be a viable and effective tool to investigate such effects, both at zero and finite temperature [12-28].

In this study we focus on the static quark-antiquark potential. The effects of the magnetic field on it have been predicted by model computations [29-33] and may have various phenomenological consequences, especially for heavy quark bound states [34-43]. Exploratory lattice results, in particular, have shown the presence of anisotropies, at zero temperature, both in the string tension and in the Coulomb coupling [21]. Our purpose is to refine such results in various directions.

At zero temperature, based on new numerical simulations, we obtain continuum extrapolated results and discuss how the potential depends on the angle between the magnetic field and the quarkantiquark separation. Then we extend our investigation to finite temperature, where we show that the

\footnotetext{
a e-mail: claudio.bonati@df.unipi.it, present address: Dipartimento di Fisica e Astronomia \& INFN-Sezione di Firenze, Via Sansone 1, 50019, Sesto Fiorentino (FI), Italy

${ }^{\mathrm{b}}$ Speaker; e-mail: massimo.delia@unipi.it

ce-mail: mariti@df.unipi.it

de-mail: mesiti@pi.infn.it

e e-mail: fnegro@pi.infn.it

fe-mail: andrea.rucci@pi.infn.it

ge-mail: f.sanfilippo@ soton.ac.uk, present address: INFN, Sez. di Roma Tre, Via della Vasca Navale 84, I-00146 Rome, Italy
} 


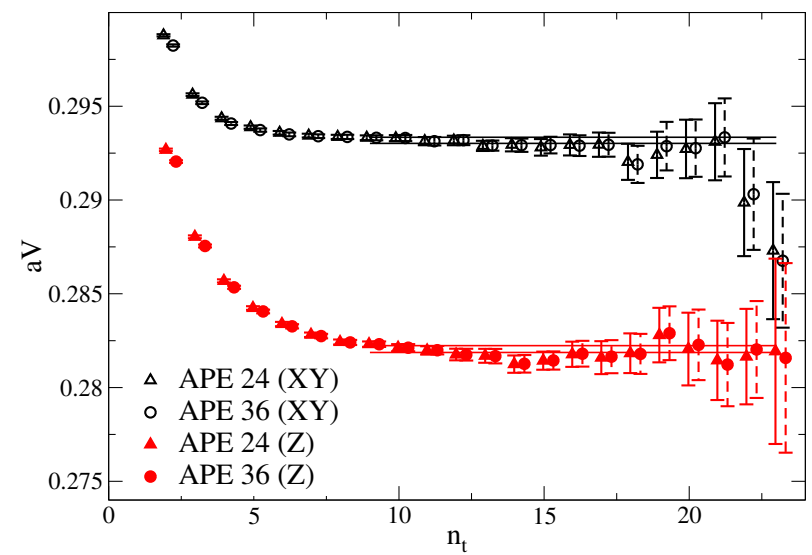

Figure 1. Results obtained for $a V_{\text {eff }}\left(a \vec{n}, a n_{t}\right)$ (see Eq. (2)) as a function of $n_{t}$ on the $48^{3} \times 96$ lattice $(a=0.0989 \mathrm{fm})$ for $|e| B \simeq 1 \mathrm{GeV}^{2}$ and two different directions of the quark-antiquark separation, transverse (XY) or longitudinal (Z) to $\vec{B}$. The quark-antiquark separation is $|\vec{n}|=3$ in lattice units, and data corresponding to two different levels of APE smearing are displayed, which are perfectly compatible with each other.

main effect of the magnetic field results in a suppression of the string tension, leading to an early loss of the confining properties of the medium, in agreement to what observed for chiral symmetry breaking [17].

We investigate $N_{f}=2+1 \mathrm{QCD}$, adopting a staggered fermion discretization with two levels of stout improving [44] and the tree level improved Symanzik action [45, 46] for the pure gauge sector. Because of the different electric charges, each quark flavor is discretized separately, exploiting the fourth root trick. We have explored 4 different lattice spacings, the smallest being $a \simeq 0.0989 \mathrm{fm}$, staying on a line of constant physics $[47,48]$ corresponding to a physical pion mass and to a strangeto-light mass ratio $m_{s} / m_{u, d}=28.15$. The spatial size has been kept equal to approximately $5 \mathrm{fm}$ for all lattice spacings. We consider a uniform magnetic field, which is introduced at the level of the discretized Dirac operator. Since we adopt, as usual, periodic boundary conditions in space, each of its components is quantized as follows:

$$
e B_{i}=6 \pi b_{i} /\left(a^{2} N_{s}^{2}\right) ; \quad b_{i} \in \mathbb{Z},
$$

where $e$ is the elementary electric charge and $N_{s}$ is the spatial extension in lattice units, which is taken equal along all directions. More technical details can be found in Ref. [49].

\section{Results at zero temperature}

At $T=0$ the static potential has been extracted from the measurements of planar temporal Wilson loops of size $\vec{n} \times n_{t}$, making use of the definition

$$
a V(a \vec{n})=\lim _{n_{t} \rightarrow \infty} a V_{\mathrm{eff}}\left(a \vec{n}, a n_{t}\right) ; \quad a V_{\mathrm{eff}}\left(a \vec{n}, a n_{t}\right) \equiv \log \left(\frac{\left\langle W\left(\vec{n}, n_{t}\right)\right\rangle}{\left\langle W\left(\vec{n}, n_{t}+1\right)\right\rangle}\right),
$$

and finding, for each fixed $\vec{n}$, a range of $n_{t}$ for which the r.h.s. of the previous definition is stable. In the presence of the external field, Wilson loops in different planes have been analyzed separately and, in 


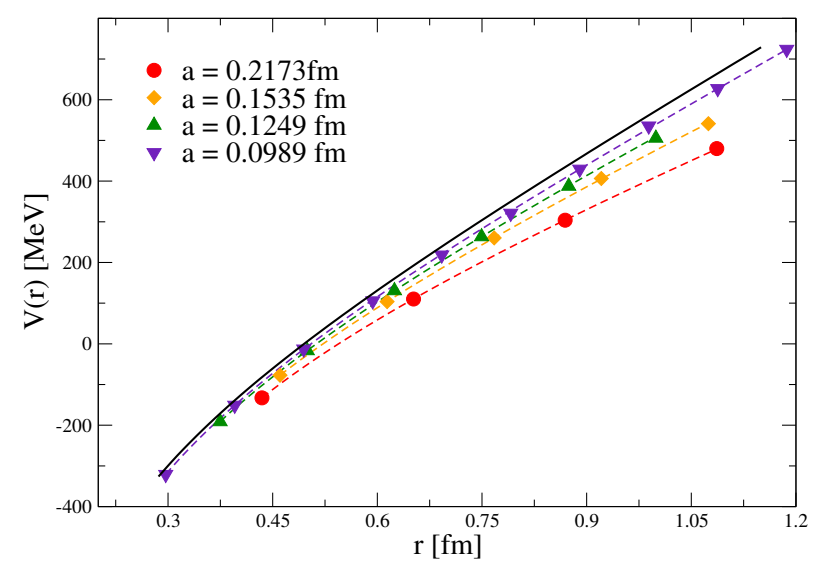

Figure 2. Static potential for various lattice spacings as a function of the quark-antiquark distance. Dashed lines correspond to a fit to the Cornell parametrization in Eq. (3). In order to permit a better comparison, for each lattice spacing the constant $V_{0}$ has been fixed so that the potential vanishes at the value of $r_{0}$ determined for that lattice. The solid line represents the continuum extrapolation.

order to reduce the UV noise, one step of HYP smearing [50, 51] and a variable number of APE smearing steps [52] (with parameter $\alpha_{\mathrm{APE}}=0.25$ ) have been adopted. An example of such procedure is shown Fig. 1. At zero external field, we can adopt the standard isotropic Cornell parametrization [53]

$$
V(r)=-\frac{\alpha}{r}+\sigma r+V_{0},
$$

where $V_{0}$ is an arbitrary constant term, $\sigma$ is the string tension and $\alpha$ is the Coulomb coupling; a related quantity is the Sommer parameter $r_{0}$ [54], defined as

$$
\left.r_{0}^{2} \frac{d V}{d r}\right|_{r_{0}}=1.65 ; \quad r_{0}=\sqrt{\frac{1.65-\alpha}{\sigma}}
$$

where last relation links $r_{0}$ to $\alpha$ and $\sigma$ in the Cornell parametrization. Results obtained for the potential at the various lattice spacings, together with a fit to Eq. (3), are reported in Fig. 2. For each lattice spacing, the constant $V_{0}$ has been fixed so that the potential vanishes at $r_{0}$. In the same figure we report a continuum extrapolation based on the three finest lattice spacings, in which we have assumed $O\left(a^{2}\right)$ corrections for each parameter (see Ref. [49] for more details); the values of the continuum extrapolated parameters are reported in Table 1.

Table 1. Continuum extrapolated values obtained for the Coulomb coupling, the string tension and the Sommer parameter.

\begin{tabular}{lll}
\hline$\alpha$ & $\sqrt{\sigma}$ & $r_{0}$ \\
\hline $0.395(22)$ & $448(20) \mathrm{MeV}$ & $0.489(20) \mathrm{fm}$ \\
\hline
\end{tabular}

At non-zero external field the potential becomes anisotropic, however results obtained in Ref. [21] have shown that, at least along the two directions parallel and orthogonal to $\vec{B}$, a Cornell parametrization still works well, even if with direction-dependent coefficients. Based on that, we assume that such 
property is maintained for an arbitrary angle $\theta$ between the magnetic field and the quark-antiquark separation (see Fig. 3) and consider the following parametrization

$$
\begin{aligned}
V(r, \theta)= & -\frac{\bar{\alpha}(B)}{r}\left(1-\sum_{n \geq 1} c_{2 n}^{\alpha}(B) \cos (2 n \theta)\right) \\
& +\bar{\sigma}(B) r\left(1-\sum_{n \geq 1} c_{2 n}^{\sigma}(B) \cos (2 n \theta)\right) \\
& +\bar{V}_{0}(B)\left(1-\sum_{n \geq 1} c_{2 n}^{V_{0}}(B) \cos (2 n \theta)\right)
\end{aligned}
$$

where the dependence of each coefficient on $\theta$ has been further expanded in a Fourier expansion.

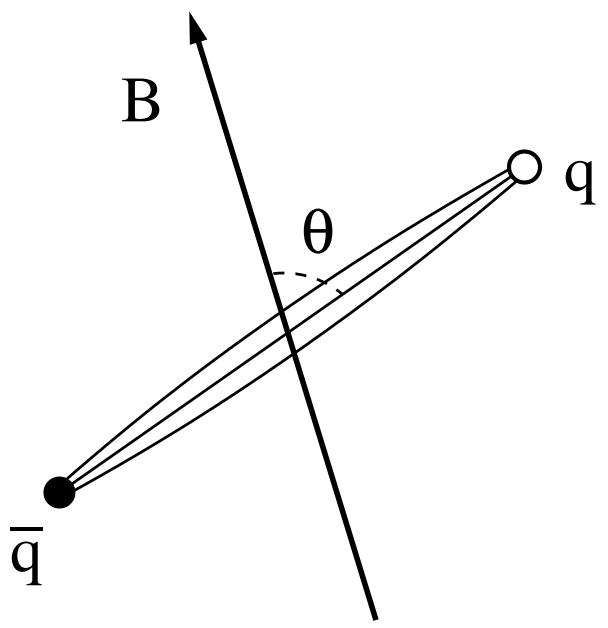

Figure 3. Relative angle $\theta$ between the magnetic field and the quark-antiquark separation. While a generic anisotropic potential could be function of the distance and of two angular variables, in the case of a uniform external field the residual rotational symmetry implies that it must be just a function of $\theta$ and $r$. Furthermore, the symmetry under inversion of $\vec{B}$ implies that $V(r, \theta)=V(r, \pi-\theta)$, leading to the general parametrization in Eq. (5).

In order to consider generic values of $\theta$ one could either rotate the Wilson loop at fixed magnetic field, or rotate $\vec{B}$ keeping the loop fixed. The first option seems cheaper, since one could perform a single Monte-Carlo simulation and measure different Wilson loops on the same sample of configurations, however it involves Wilson loops which are not parallel to any of the lattice axes and have different renormalization properties. For that reason, we have chosen the second option, even if the need for new simulations has limited our range of exploration. We have considered two lattice spacings, $a=0.0989$ and $0.1535 \mathrm{fm}$, with lattices of size $48^{3} \times 96$ and $32^{4}$ respectively. In both cases we have considered the following magnetic field quanta: $\left(b_{x}, b_{y}, b_{z}\right)=(0,0,32),(4,13,29)$ and $(9,18,25)$, all of which correspond to $|e| B \sim 1 \mathrm{GeV}^{2}$; considering Wilson loops in the three different temporal planes, that gives us access to 8 different values of the angle $\theta$.

Results obtained at the finer lattice spacing are shown in Fig. 4. Consistently with the results of Ref. [21], we observe that, at fixed $r$, the potential increases as $\theta$ increases, reaching a maximum for $\theta=\pi / 2$. Moreover, as one tries to perform a best fit according to Eq. (5), one finds that the inclusion of the lowest order coefficient $c_{2}$, for each parameter, is sufficient to provide a good description of all data. The best fit function is displayed in Fig. 4 as well.

The fact that the angular dependence can be described, at least within our present statistical errors, by the first non-trivial harmonic term, implies that all relevant parameters can be determined by an analysis of the potential along the directions orthogonal and parallel to $\vec{B}$. For that reason, further simulations have been limited to $\vec{B}$ directed along the $\hat{z}$ axis. In particular, for each parameter $O$, we 


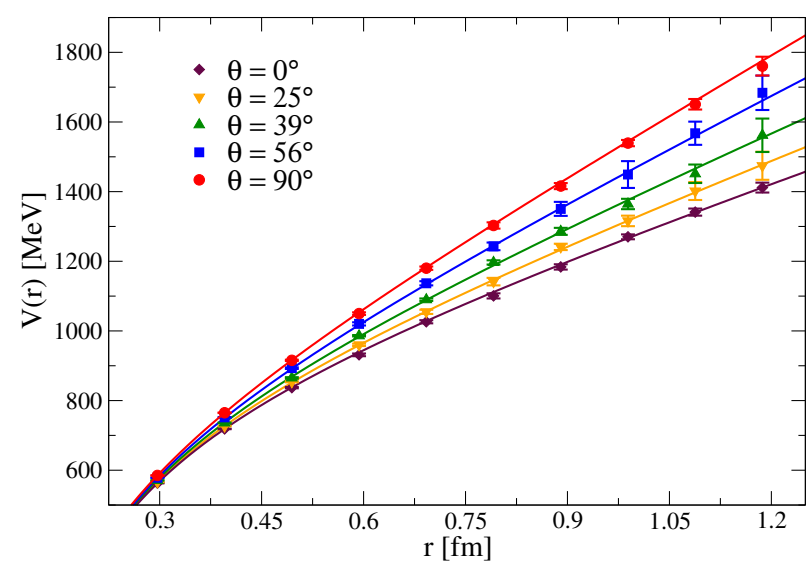

Figure 4. Values of the potential, extracted using Eq. (2), obtained on the $48^{3} \times 96$ lattice, for $|e| B \simeq 1 \mathrm{GeV}^{2}$ and for different values of the angle $\theta$. The solid lines correspond to a global best fit according to Eq. (5), setting $c_{2 n}=0$ for $n>1$.
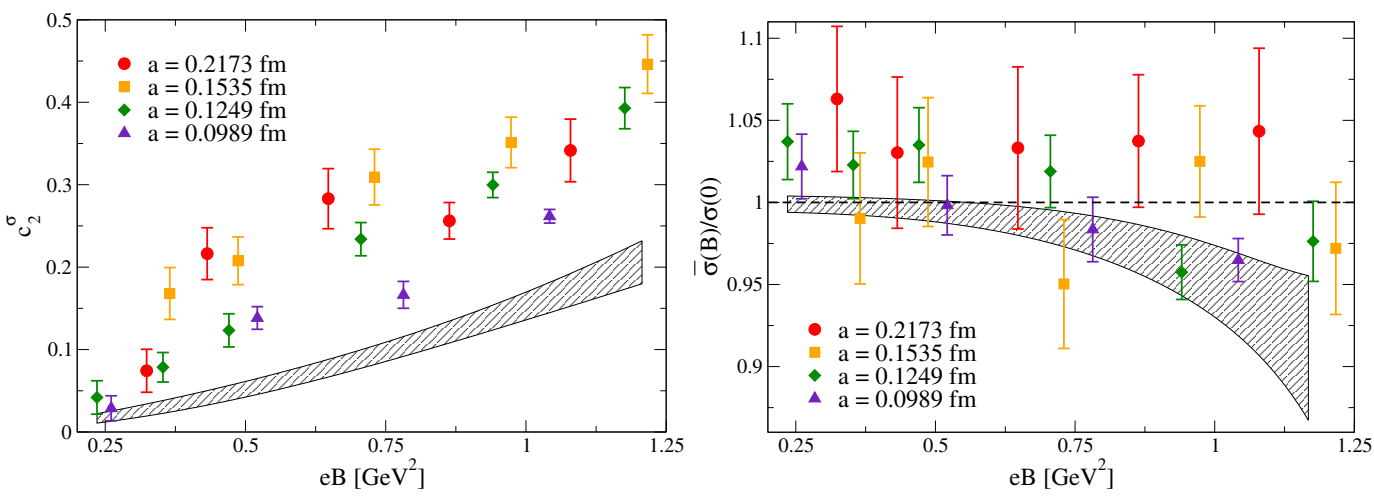

Figure 5. String tension parameters for different values of the lattice spacing, see Eqs. (6) and (7). Gray bands represent the continuum extrapolation, see Eq. (8), obtained using all data except those from the coarsest lattice.

can introduce the anisotropy

$$
\delta^{O}(|e| B)=\frac{O_{X Y}(|e| B)-O_{Z}(|e| B)}{O_{X Y}(|e| B)+O_{Z}(|e| B)}=\sum_{n} c_{2 n}^{O} \simeq c_{2}^{O}
$$

and the average relative change

$$
R^{O}(|e| B)=\frac{O_{X Y}(|e| B)+O_{Z}(|e| B)}{2 O(|e| B=0)}=\frac{\bar{O}(|e| B)}{O(|e| B=0)}\left(1-\sum_{n \text { even }} c_{2 n}^{O}\right) \simeq \frac{\bar{O}(|e| B)}{O(|e| B=0)}
$$

where in both cases last equalities hold under the assumption $c_{2 n} \simeq 0$ for $n>1$. Results obtained in this way are shown, for the case of the string in tension, in Fig. 5. A continuum extrapolation has then 


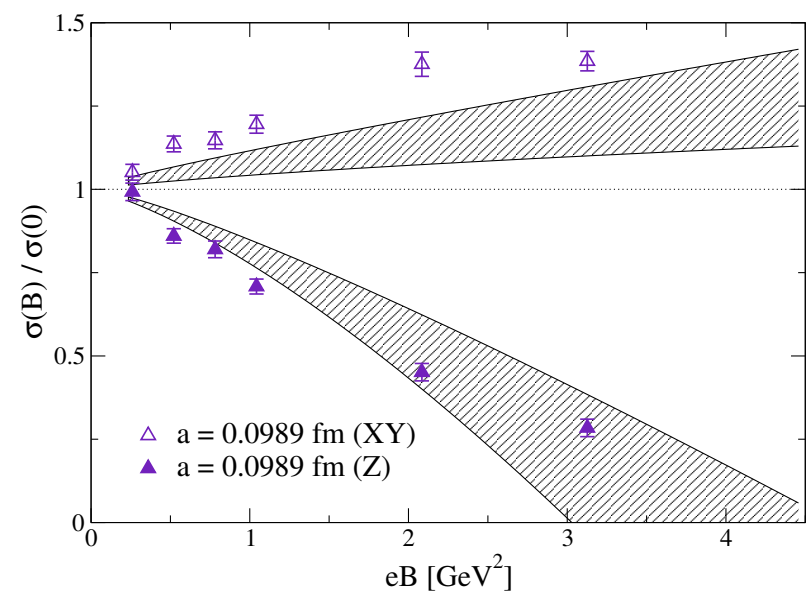

Figure 6. Relative change of $\sigma$, as a function of $|e| B$, along the longitudinal and trasverse directions. The shadowed band corresponds to the continuum limit, which however has been obtained based on results at $|e| B \lesssim 1$ $\mathrm{GeV}^{2}$.

been performed, according to the following ansatz

$$
\begin{aligned}
& c_{2}^{O}=A^{O}\left(1+C^{O} a^{2}\right)(|e| B)^{D^{O}\left(1+E^{O} a^{2}\right)} \\
& R^{O}=1+\bar{A}^{O}\left(1+\bar{C}^{O} a^{2}\right)(|e| B)^{\bar{D}^{O}}
\end{aligned}
$$

Reasonable fits are obtained by considering only data from the three finest lattices. The results obtained for the continuum extrapolation can be summarized as follows. The anisotropy of the string tension has a well defined non-zero continuum limit and is of the order of $15 \%$ for $|e| B \sim 1 \mathrm{GeV}^{2}$; on the contrary, those of the Coulomb term and of $V_{0}$ are compatible with zero. The ratios $R^{O}$ instead show little or negligible dependence on the magnetic field for all parameters. Therefore, whereas the effects of the magnetic field on the quark-antiquark potential persist in the continuum limit, they seem to be simply related to the anisotropy induced at the level of string tension.

It is interesting to further investigate what happens, due to this anisotropy, as the magnetic field becomes very large. Continuum results obtained for the string tension are shown in Fig. 6 in an extended range of magnetic fields, and suggest that the longitudinal string tension could vanish for $e B \sim 4 \mathrm{GeV}^{2}$. Of course it is not clear how reliable such prediction could be, since our continuum extrapolation is based on results obtained in a much restricted range of magnetic fields, going up to $1 \mathrm{GeV}^{2}$. To have more reliable indications, we have performed new numerical simulations at larger values of $|e| B$, precisely at $|e| B \sim 2$ and $3 \mathrm{GeV}^{2}$, however this has been possible only on the finest lattice $a=0.0989 \mathrm{fm}$, where cut-off effect should still be under control, even at this large value of $B$. The new results are shown in Fig. 6 and suggest that the steady decrease of longitudinal string tension continues up to very large values of the magnetic field, so that its vanishing at some critical value of $B$ is a realistic possibility. On the other hand, a quantitative confirmation of that requires further dedicated simulations on finer lattices, that we plan to perform in future investigations. 

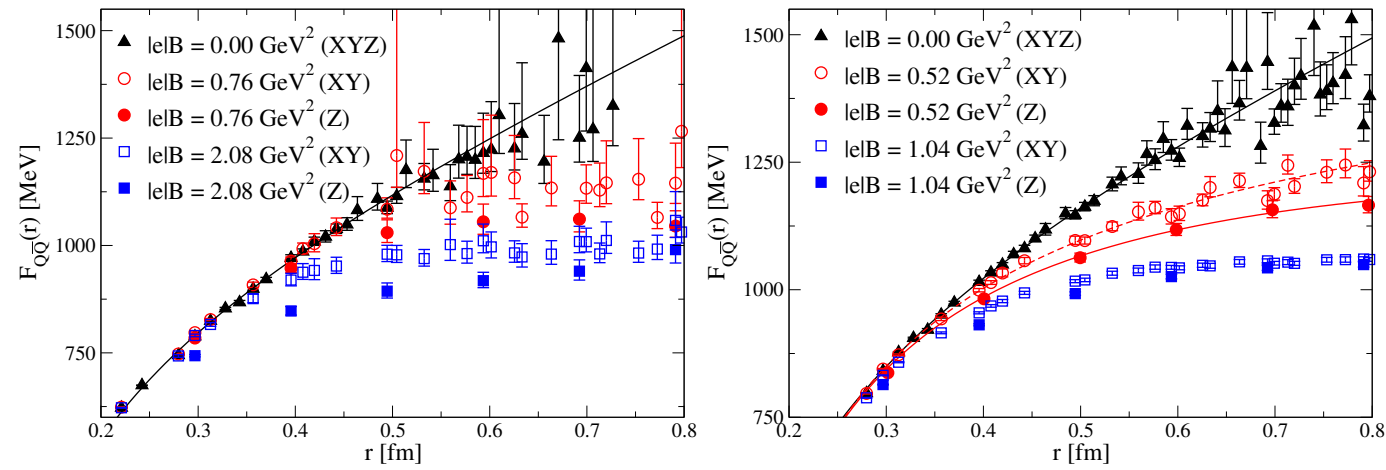

Figure 7. Free energy of the $Q \bar{Q}$ pair, $F_{Q \bar{Q}}(r, T)$ at $T \simeq 100 \mathrm{MeV}$ (left) and $T \simeq 125 \mathrm{MeV}$ (right) for several values of the magnetic field. Continuum and dashed lines correspond to best fits to a Cornell parametrization, and are reported only when the best fit works well in some range of distances.

\subsection{Results at finite temperature}

At finite $T$, the static potential has been determined from Polyakov loop correlators

$$
C(\vec{n}, T)=\left\langle\operatorname{Tr} L(\vec{r}) \operatorname{Tr} L^{\dagger}(\vec{r}+\vec{n})\right\rangle
$$

where $\vec{r}$ and $\vec{n}$ are dimensionless lattice vectors. This observable is related to the free energy $F_{Q \bar{Q}}(a \vec{n}, T)$ of a static quark-antiquark pair, and can be shown to receive contributions only from the singlet channel [55-60]. Therefore, we adopt the definition

$$
F_{Q \bar{Q}}(a \vec{n}, T)=-\frac{1}{a N_{t}} \log C(\vec{n}, T)
$$

which, apart from $T$-dependent additive renormalizations, we take as an estimate of the static quarkantiquark potential at finite $T$.

We report results at a single value of the lattice spacing, $a \simeq 0.0989 \mathrm{fm}$, and for three temperatures below $T_{c}, T \simeq 100,125,143 \mathrm{MeV}$ (corresponding to $48^{3} \times 20,16,14$ lattices). In Fig. 7 we show the results obtained for $F_{Q \bar{Q}}$ at some values of the magnetic field, respectively for $T \simeq 100 \mathrm{MeV}$ and $T \simeq 125 \mathrm{MeV}$.

The anisotropy is still visible, however the main effect of the magnetic field seems to be a suppression of the potential in all directions, so that for large enough fields the potential cannot be fitted according to a Cornell parametrization, something that we interpret in term of a vanishing string tension. The observed behavior is consistent with the decrease of the chiral pseudocritical temperature which has been observed in previous studies $[15,17]$.

The observed suppression of the confining properties of the medium, even at moderately low values of $T$, induced by $B$ could have various interesting interpretations and consequences. First, one could interpret the decrease of $T_{c}$ as a function of $B$ in terms of a "deconfinement catalysis", a point of view which is suggested also by recent model computations [61-63]. Moreover, the modification of the confining properties might have important consequences for the suppression of heavy quark bound states in the thermal medium produced in non-central heavy ion collisions. 


\section{Acknowledgments}

We acknowledge PRACE for awarding us access to the resources of the Blue Gene/Q machine FERMI, based in Italy at CINECA, under project Pra09-2400 - SISMAF. FS received funding from the European Research Council, under the European Community Seventh Framework Programme (FP7/2007-2013) ERC grant agreement No 279757. FN acknowledges financial support from the INFN SUMA project.

\section{References}

[1] V. Skokov, A. Y. Illarionov and V. Toneev, Int. J. Mod. Phys. A 24, 5925 (2009) [arXiv:0907.1396 [nucl-th]].

[2] V. Voronyuk, V. D. Toneev, W. Cassing, E. L. Bratkovskaya, V. P. Konchakovski and S. A. Voloshin, Phys. Rev. C 83, 054911 (2011) [arXiv:1103.4239 [nucl-th]].

[3] A. Bzdak and V. Skokov, Phys. Lett. B 710, 171 (2012) [arXiv:1111.1949 [hep-ph]].

[4] W. -T. Deng and X. -G. Huang, Phys. Rev. C 85, 044907 (2012) [arXiv:1201.5108 [nucl-th]].

[5] K. Tuchin, Adv. High Energy Phys. 2013, 490495 (2013) [arXiv:1301.0099].

[6] R. Holliday and K. Tuchin, arXiv:1604.04572 [hep-ph].

[7] T. Vachaspati, Phys. Lett. B 265, 258 (1991).

[8] D. Grasso and H. R. Rubinstein, Phys. Rept. 348, 163 (2001) [astro-ph/0009061].

[9] R. C. Duncan and C. Thompson, Astrophys. J. 392, L9 (1992).

[10] D. E. Kharzeev, K. Landsteiner, A. Schmitt and H. U. Yee, Lect. Notes Phys. 871, 1 (2013).

[11] V. A. Miransky and I. A. Shovkovy, Phys. Rept. 576, 1 (2015) [arXiv:1503.00732 [hep-ph]].

[12] M. D’Elia, S. Mukherjee and F. Sanfilippo, Phys. Rev. D 82, 051501 (2010) [arXiv:1005.5365 [hep-lat]].

[13] P. V. Buividovich, M. N. Chernodub, D. E. Kharzeev, T. Kalaydzhyan, E. V. Luschevskaya and M. I. Polikarpov, Phys. Rev. Lett. 105, 132001 (2010) [arXiv:1003.2180 [hep-lat]].

[14] M. D’Elia and F. Negro, Phys. Rev. D 83, 114028 (2011) [arXiv:1103.2080 [hep-lat]].

[15] G. S. Bali, F. Bruckmann, G. Endrodi, Z. Fodor, S. D. Katz, S. Krieg, A. Schafer and K. K. Szabo, JHEP 1202, 044 (2012) [arXiv:1111.4956 [hep-lat]].

[16] E.-M. Ilgenfritz, M. Kalinowski, M. Muller-Preussker, B. Petersson and A. Schreiber, Phys. Rev. D 85, 114504 (2012) [arXiv:1203.3360 [hep-lat]].

[17] G. S. Bali, F. Bruckmann, G. Endrodi, Z. Fodor, S. D. Katz and A. Schafer, Phys. Rev. D 86, 071502 (2012) [arXiv:1206.4205 [hep-lat]].

[18] M. D’Elia, M. Mariti and F. Negro, Phys. Rev. Lett. 110, 082002 (2013) [arXiv:1209.0722 [heplat]].

[19] F. Bruckmann, G. Endrodi and T. G. Kovacs, JHEP 1304, 112 (2013) [arXiv:1303.3972 [heplat]].

[20] E.-M. Ilgenfritz, M. Muller-Preussker, B. Petersson and A. Schreiber, Phys. Rev. D 89, 054512 (2014) [arXiv:1310.7876 [hep-lat]].

[21] C. Bonati, M. D'Elia, M. Mariti, M. Mesiti, F. Negro and F. Sanfilippo, Phys. Rev. D 89, 114502 (2014) [arXiv:1403.6094 [hep-lat]].

[22] A. Schäfer, G. Endrodi, and J. Wellnhofer, Phys. Rev. D 92, 1, 014509 (2015) [arXiv:1506.07698 [hep-lat]].

[23] M. D’Elia, E. Meggiolaro, M. Mesiti and F. Negro, Phys. Rev. D 93, 054017 (2016) [arXiv:1510.07012 [hep-lat]]. 
[24] C. Bonati, M. D’Elia, M. Mariti, F. Negro and F. Sanfilippo, Phys. Rev. D 89, 054506 (2014) [arXiv:1310.8656 [hep-lat]]; Phys. Rev. Lett. 111, 182001 (2013) [arXiv:1307.8063 [hep-lat]]. [25] L. Levkova and C. DeTar, Phys. Rev. Lett. 112, 012002 (2014) [arXiv:1309.1142 [hep-lat]].

[26] G. S. Bali, F. Bruckmann, G. Endrodi, F. Gruber and A. Schaefer, JHEP 1304, 130 (2013) [arXiv:1303.1328 [hep-lat]].

[27] G. S. Bali, F. Bruckmann, G. Endrodi, S. D. Katz and A. Schaefer, JHEP 1408, 177 (2014) [arXiv:1406.0269 [hep-lat]].

[28] P. Cea and L. Cosmai, JHEP 1512, 058 (2015) [arXiv:1509.01982 [hep-lat]].

[29] V. A. Miransky and I. A. Shovkovy, Phys. Rev. D 66, 045006 (2002) [hep-ph/0205348].

[30] M. N. Chernodub, Mod. Phys. Lett. A 29, 1450162 (2014).

[31] R. Rougemont, R. Critelli and J. Noronha, Phys. Rev. D 91, 066001 (2015) [arXiv:1409.0556 [hep-th]].

[32] E. J. Ferrer, V. de la Incera and X. J. Wen, Phys. Rev. D 91, 054006 (2015) [arXiv:1407.3503 [nucl-th]].

[33] Y. A. Simonov and M. A. Trusov, Phys. Lett. B 747, 48 (2015) [arXiv:1503.08531 [hep-ph]].

[34] J. Alford and M. Strickland, Phys. Rev. D 88, 105017 (2013) [arXiv:1309.3003 [hep-ph]].

[35] D. Dudal and T. G. Mertens, Phys. Rev. D 91, 086002 (2015) [arXiv:1410.3297 [hep-th]].

[36] S. Cho, K. Hattori, S. H. Lee, K. Morita and S. Ozaki, Phys. Rev. D 91, 045025 (2015) [arXiv:1411.7675 [hep-ph]].

[37] H. Taya, Phys. Rev. D 92, 014038 (2015) [arXiv:1412.6877 [hep-ph]].

[38] C. Bonati, M. D’Elia and A. Rucci, Phys. Rev. D 92, 054014 (2015) [arXiv:1506.07890 [hep$\mathrm{ph}]$.

[39] K. Hattori, T. Kojo and N. Su, Nucl. Phys. A 951, 1 (2016) [arXiv:1512.07361 [hep-ph]].

[40] K. Suzuki and T. Yoshida, Phys. Rev. D 93, 051502 (2016) [arXiv:1601.02178 [hep-ph]]; arXiv:1607.04935 [hep-ph].

[41] X. Guo, S. Shi, N. Xu, Z. Xu and P. Zhuang, Phys. Lett. B 751, 215 (2015) [arXiv:1502.04407 [hep-ph]].

[42] K. Fukushima, K. Hattori, H. U. Yee and Y. Yin, Phys. Rev. D 93, 074028 (2016) [arXiv:1512.03689 [hep-ph]].

[43] P. Gubler, K. Hattori, S. H. Lee, M. Oka, S. Ozaki and K. Suzuki, Phys. Rev. D 93, 054026 (2016) [arXiv:1512.08864 [hep-ph]].

[44] C. Morningstar and M. J. Peardon, Phys. Rev. D 69, 054501 (2004) [hep-lat/0311018].

[45] P. Weisz, Nucl. Phys. B 212, 1 (1983).

[46] G. Curci, P. Menotti and G. Paffuti, Phys. Lett. B 130, 205 (1983) [Erratum-ibid. B 135, 516 (1984)].

[47] Y. Aoki, S. Borsanyi, S. Durr, Z. Fodor, S. D. Katz, S. Krieg and K. K. Szabo, JHEP 0906, 088 (2009) [arXiv:0903.4155 [hep-lat]].

[48] S. Borsanyi, G. Endrodi, Z. Fodor, A. Jakovac, S. D. Katz, S. Krieg, C. Ratti and K. K. Szabo, JHEP 1011, 077 (2010) [arXiv:1007.2580 [hep-lat]].

[49] C. Bonati, M. D’Elia, M. Mariti, M. Mesiti, F. Negro, A. Rucci and F. Sanfilippo, Phys. Rev. D 94, no. 9, 094007 (2016) [arXiv:1607.08160 [hep-lat]].

[50] A. Hasenfratz and F. Knechtli, Phys. Rev. D 64, 034504 (2001) [hep-lat/0103029].

[51] M. Della Morte, A. Shindler and R. Sommer, JHEP 0508, 051 (2005) [hep-lat/0506008].

[52] M. Albanese et al. [APE Collaboration], Phys. Lett. B 192 (1987) 163. 
[53] E. Eichten, K. Gottfried, T. Kinoshita, J. B. Kogut, K. D. Lane and T. M. Yan, Phys. Rev. Lett. 34, 369 (1975) [Erratum-ibid. 36, 1276 (1976)].

[54] R. Sommer, Nucl. Phys. B 411, 839 (1994) [hep-lat/9310022].

[55] L. S. Brown and W. I. Weisberger, Phys. Rev. D 20, 3239 (1979).

[56] L. D. McLerran and B. Svetitsky, Phys. Rev. D 24, 450 (1981).

[57] S. Nadkarni, Phys. Rev. D 34, 3904 (1986).

[58] O. Kaczmarek, F. Karsch, P. Petreczky and F. Zantow, Phys. Lett. B 543, 41 (2002) [heplat/0207002].

[59] O. Jahn and O. Philipsen, Phys. Rev. D 70, 074504 (2004) [hep-lat/0407042].

[60] G. Rossi and M. Testa, Phys. Rev. D 87, 085014 (2013) [arXiv:1304.2542 [hep-lat]].

[61] E. S. Fraga and L. F. Palhares, Phys. Rev. D 86, 016008 (2012) [arXiv:1201.5881 [hep-ph]].

[62] E. S. Fraga, J. Noronha and L. F. Palhares, Phys. Rev. D 87, 114014 (2013) [arXiv:1207.7094 [hep-ph]].

[63] D. Dudal, D. R. Granado and T. G. Mertens, Phys. Rev. D 93, 125004 (2016) [arXiv:1511.04042 [hep-th]]. 